\title{
A new strategy for helioseismic inversions
}

\author{
A. Eff-Darwich and F. Pérez Hernández \\ Instituto de Astrofísica de Canarias, E-38205 La Laguna, Tenerife, Spain
}

Received April 11; accepted December 20, 1996

\begin{abstract}
Helioseismic inversion techniques have been revealed as powerful tools for inferring the internal structure and dynamics of the Sun. One of the most popular techniques is Regularized Least Squares. When it is used, it is necessary to define an inversion mesh and a penalty function, without an a priori knowledge of the behaviour of the solution. In addition, this penalty function is weighted by a trade-off parameter that must be fixed in order to obtain the solution.

We present here a new technique, developed in order to find the optimal mesh and smoothing function by means of a deep analysis of the basis functions of the inversion problem. We have found that the method is suitable in particular for obtaining the sound speed and density profiles simultaneously, without any reference to the equation of state.
\end{abstract}

Key words: methods: numerical — Sun: oscillations Sun: interior

\section{Introduction}

A typical one-dimensional (radial) inversion problem, is given by the following integral relation

$d_{i}=\int_{0}^{R} K_{i}(r) f(r) \mathrm{d} r+\epsilon_{i}$,

where $d_{i}$ are the data available with error $\epsilon_{i}$. The function $f(r)$, where $r$ is the independent spatial variable, is the one we want to obtain by inverting relation (1). The basis functions $K_{i}(r)$, called kernels, are known functions that give the contribution of each radial point to the value of the data $d_{i}$. In helioseismic inversion problems, $d_{i}$ could be the frequency differences between a solar model and the actual Sun, then $f(r)$ is related to the difference in some structural parameter between the Sun and the solar model. On the other hand, if $d_{i}$ are rotational splittings, or any combination of them, then $f(r)$ gives information about the solar rotational rate.

Send offprint requests to: A. Eff-Darwich
Inversion of relation (1) is an ill-posed problem (Thompson 1995), hence the solution is unstable and shows undesired high-frequency oscillations that must be avoided. This problem has been solved by several methods that can be classified in two different techniques: Regularized Least Squares (RLS, Craig \& Brown 1986) and Optimal Localized Averages (OLA, Backus \& Gilbert $1968,1970)$. Both give the solution as a linear combination of the data, but in a different way.

RLS requires the discretization of the integral relation to be inverted. In our case, Eq. (1) is transformed into a matrix relation

$D=A f+\Sigma$,

where $D$ is the data vector, with elements $d_{i}$ and dimension $M, f$ is the solution vector to be determined at $N$ tabular points, $A$ is the matrix with the kernels, of dimension $M \times N$, and $\Sigma$ is the vector containing the errors in D.

The RLS solution is the one that minimizes the quadratic difference $\chi^{2}=|A f-D|^{2}$, with a constraint given by a smoothing matrix, $H$, introduced in order to avoid the instabilities in the solution. The general relation to be minimized is

$s(f)=(A f-D)^{\mathrm{T}}(A f-D)+\gamma f H f$

where $\gamma$ is a escalar introduced to give a suitable weight to the constraint matrix $H$ in the solution. Hence, the function $f$ is approximated by

$f^{\text {est }}=\left(A^{\mathrm{T}} A+\gamma H\right)^{-1} A^{\mathrm{T}} D$

while the error $\varepsilon^{\text {est }}$ is calculated as

$\varepsilon^{\mathrm{est}}=\left(A^{\mathrm{T}} A+\gamma H\right)^{-1} A^{\mathrm{T}} \Sigma$.

In general, the weighting factor $\gamma$, as well as the form of the smoothing condition $H$ must be choosen before the inversion is done, without an a priori knowledge of the behaviour of the solution. Something similar happens with the number and distribution of points in the inversion mesh. These must be fixed in a previous step in the inversion procedure, without any information of how optimal 
such a mesh is. Therefore, there is some degree of arbitrariness in the procedure that can lead to significantly different solutions.

The OLA technique is more sophisticated and tries to obtain, at any point $r_{0}$ of the mesh, a combination of the kernels (with coefficients $q_{i}, i=1, \cdots, M$ ), called the averaged kernel $\mathcal{K}\left(r_{0}, r\right)$, that mimics a $\delta$-function around $r_{0}$, but with a moderate error propagation. With such an averaged kernel, it is possible to calculate the solution as

$f\left(r_{0}\right)=\int_{0}^{R} \mathcal{K}\left(r_{0}, r\right) f(r) \mathrm{d} r=\sum_{i=1}^{M} q_{i} d_{i}$

It is necessary to include a parameter (analogous to the weight of the smoothing function in RLS) that is a tradeoff between the "Dirac delta behaviour" of $\mathcal{K}\left(r_{0}, r\right)$ and its error propagation. The main problem with this method is in choosing the trade-off parameter. When dealing with two functions $f_{1}(r)$ and $f_{2}(r)$ (for instance sound speed and density), and hence two trade-off parameters, this method is particularly problematical.

\section{A new method: Optimal Mesh Distribution (OMD)}

We have already pointed out the main problems when helioseismic data are inverted with standard techniques. The method we have developed, called Optimal Mesh Distribution (OMD), is a mixture of both the aforementioned techniques, although it is based mainly on RLS.

OMD tries to solve the main disadvantages of RLS by taking care of looking for the optimal mesh and defining the smoothing function by a deep analysis of the properties of the basis function of the problem. In particular, a different smoothness to the solution at each point of the mesh is used.

In order to illustrate the method developed here, we will take as $d_{i}$ the rotational splittings parameterized as Clebsh-Gordan coefficients, so $f(r)$ will be a measurement of the solar rotational rate (e.g. Ritzwoller \& Lavely 1991). To test the method, a set of artificial data has been created. These consist in $s=1$ Clebsh-Gordan coefficients, obtained from the rotation curve shown in Fig. 6, for 1380 p-modes with $l$ ranging from 1 to 150 and frequencies in the interval $1500 \mu \mathrm{Hz} \leq \nu \leq 4500 \mu \mathrm{Hz}$. Noise and error levels are taken from actual observations. We will call this set test 1 .

In Sect. 3 we will apply the method to a more complex problem, where instead of one function $f(r)$, we work with two, sound speed and density.

\subsection{Determination of the mesh}

The radial resolution that can be achieved in a given inverse problem depends on the mode data set used through the associated kernels. Since the kernels scan the solar interior in a significantly non-homogeneous way, the resolution strongly depends on $r$. In fact, one of the main problems when a solution is obtained, is that it can show oscillations due to an excessive number of points in the mesh. But then, it seems a good idea to decrease the number of points according to the spatial resolution; that is, we will have a non-equally spaced mesh point distribution.

The basic idea in obtaining the spatial resolution is as follows. Since the result is obtained by means of linear combinations of kernels, the solution at a given radial point can contain oscillatory patterns only with larger periods than those given for such a linear combination. Thus, in the procedure we will consider a given spatial oscillatory pattern (a sinusoidal function) and search for the combination of kernels that better fit this behaviour. If the fit is good at a given radial point, then the solution at this point is allowed for having such an oscillatory pattern. The smallest period for which a good fit is obtained at a given $r$ gives the cut-off spatial frequency, and hence the spatial resolution. Finally, the mesh is built by taking only one radial point within the interval given by the spatial resolution.

To calculate the spatial resolution given by the kernels, a variant of the OLA method has been developed. As a measurement of the width of the averaging kernel, OLA uses a quadratic form. A variant of OLA, called SOLA (Pijpers \& Thompson 1994), uses the quadratic deviation of the combination of kernels from a "target" function, in the ideal case a Dirac delta function. In our case, the "target" functions are sine waves of different frequencies; namely,

$\Phi(r)=a \cdot \sin \left(\omega_{\Phi} r\right)$,

where $\omega_{\Phi}$ is the spatial frequency. The amplitude $a$ is obtained by assigning the area of the sine function as unity.

Let us call $b$ the coefficients of the linear combination of kernels, the vector of dimension $M$ we want to determine with this analysis. We want to minimize the quantity

$\int_{0}^{R}\left(b^{\mathrm{T}} P(r)-\Phi(r)\right)^{2} \mathrm{~d} r$,

where the vector $P$ is defined by

$P_{i}(r)=K_{i}(r) \quad i=1, \ldots, M$

The corresponding normal Gaussian equations are

$C b=y$,

where $C$ is the kernel's covariance matrix of dimensions $M \times M$, with elements $c_{i j}$ given by

$c_{i j}=\int_{0}^{R} K_{i} K_{j} \mathrm{~d} r$ 
and $y$ is the "target" function vector,

$y_{i}=\int_{0}^{R} K_{i}(r) \Phi(r) \mathrm{d} r$.

Then the unknown vector $b$ can be obtained by inverting relation (10). Since $C$ is a quasi-singular matrix, we obtain $b$ as in the RLS technique, by keeping $|C b-y|^{2} \leq \mathrm{e}^{2}$ and imposing the constraint $b^{\mathrm{T}} b=$ constant, weighted by a parameter $\lambda$, i.e. we minimize the quadratic form $s$ defined by

$s(b)=(C b-y)^{\mathrm{T}}(C b-y)+\lambda b^{\mathrm{T}} b$.

Therefore, the estimated solution is given by

$b=\left(C^{\mathrm{T}} C+\lambda I\right)^{-1} C^{\mathrm{T}} y$.

The parameter $\lambda$ is introduced in order to avoid solutions with undesirable oscillatory components. Nevertheless, its value is very small, and there are a wide range of values of $\lambda$ for which the fit to the target function is almost invariant and very good.

Let we consider test 1. As illustrative cases, Figs. $1 \mathrm{a}$ and $1 \mathrm{~b}$ show the best fit of the kernel combinations $\sum_{i} b_{i} K_{i}$, for two values of $1 / \omega_{\Phi}: 0.018 R$ and $0.11 R$. The sine with spatial period of $0.11 R$ is well fitted by the combination of kernels at almost all the radial points. However for a period of $0.11 R$, only points with $r / R>0.75$ can be fitted properly.
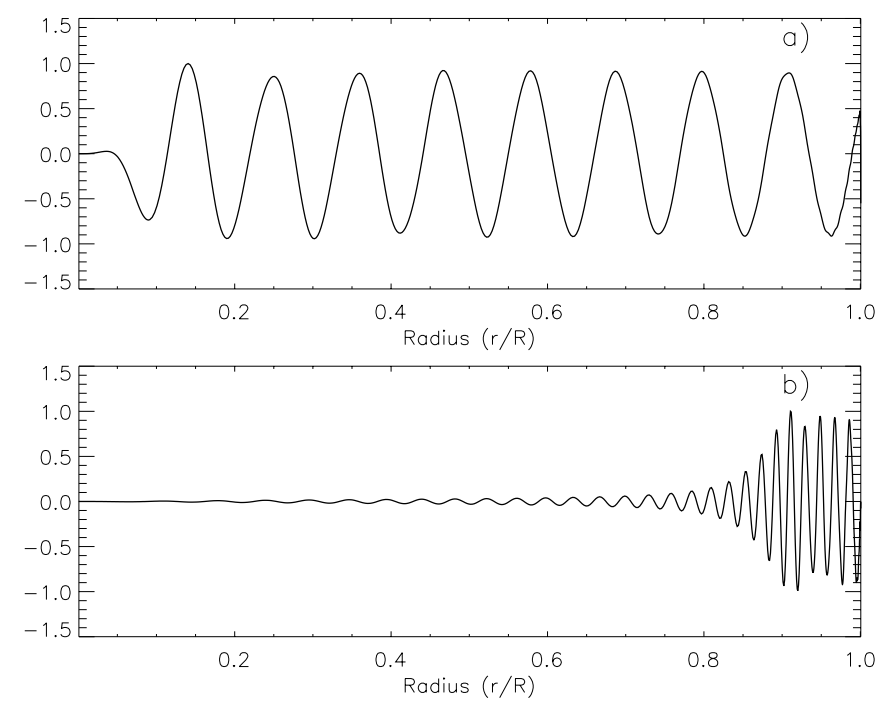

Fig. 1. a) Best fit to a sine with period $0.11 R$. b) The same but for a period of $0.018 R$. test 1 has been considered

Once $b$ is obtained for a given spatial frequency $\omega_{\Phi}$, we need to define the goodness of the fit quantitatively. To do this, we compute the quadratic deviation $\chi^{2}\left(r_{i}\right)$ as a function of the radial point $r_{i}$ and for a given $\omega_{\Phi}$ :

$\chi^{2}\left(r_{i}\right)=\int_{r_{1, i}}^{r_{2, i}}\left(b^{\mathrm{T}} P(r)-\Phi(r)\right)^{2} \mathrm{~d} r$ where $r_{2, i}-r_{1, i}=2 / \omega_{\Phi}$ and $r_{i}=\left(r_{1, i}+r_{2, i}\right) / 2$.

In Fig. 2 is represented the value of $\chi\left(r_{i}\right)$ as a function of the spatial period $1 / \omega_{\Phi}$, and for two points with $r_{i}=$ $0.3 R$ and $r_{i}=0.61 R$. The fit is good for values of $\omega_{\Phi}$ for which $\chi^{2}$ is smaller than a given tolerance factor $\epsilon$. In particular, we have taken $\epsilon=0.5$, but as can be seen in Fig. 2, there is a sharp change in the behaviour of $\chi^{2}\left(r_{i}\right)$, so there is a wide range of values for $\epsilon$ for which the result remains almost unchanged.

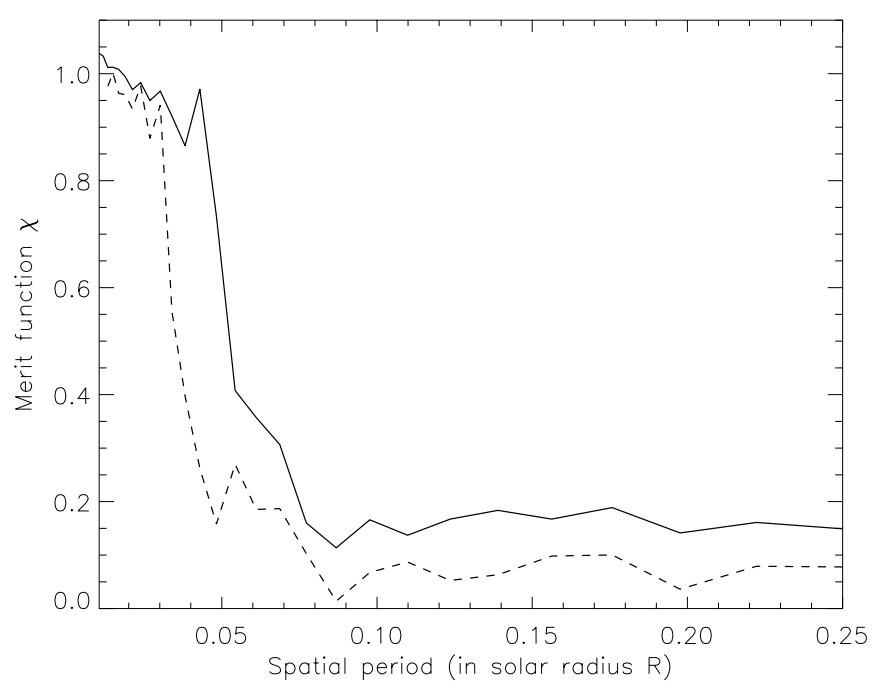

Fig. 2. $\chi\left(r_{i}\right)$ as a function of spatial period $1 / \omega_{\Phi}$ for $r_{i}=0.3 R$ (solid line) and for $r_{i}=0.61 R$ (dashed lines)

We define the cut-off frequency as the value of $\omega_{\Phi}$ for which $\chi^{2} \simeq \epsilon$. The spatial resolution at a point $r_{i}$ is defined as the inverse of the cut-off frequency. Figure 3 shows the spatial resolution obtained for test 1 . Since only $p$ modes are used, the resolution is worse at deeper points.

We can now build the inversion mesh by including only one radial point within the interval given by the spatial resolution. The resulting number of points is too low, in particular for test 1 we have $N=89$. Therefore the discretisation of relation (1) could not be accurate enough. To avoid this problem, we have increased the actual spatial resolution by a factor of four, so that the number of points in the mesh grows in the same amount. With such a mesh, the discretization is very good, but some oscillations will appear in the solution.

\subsection{Radial dependence of the smoothing function}

As in the RLS method, to avoid oscillations in the solution, it is necessary to define and give a weight to the smoothing constraint. The other novelty of OMD is the way the smoothing function is defined directly from the spatial resolution analysis, and how it is weighted differently for each radial point. 


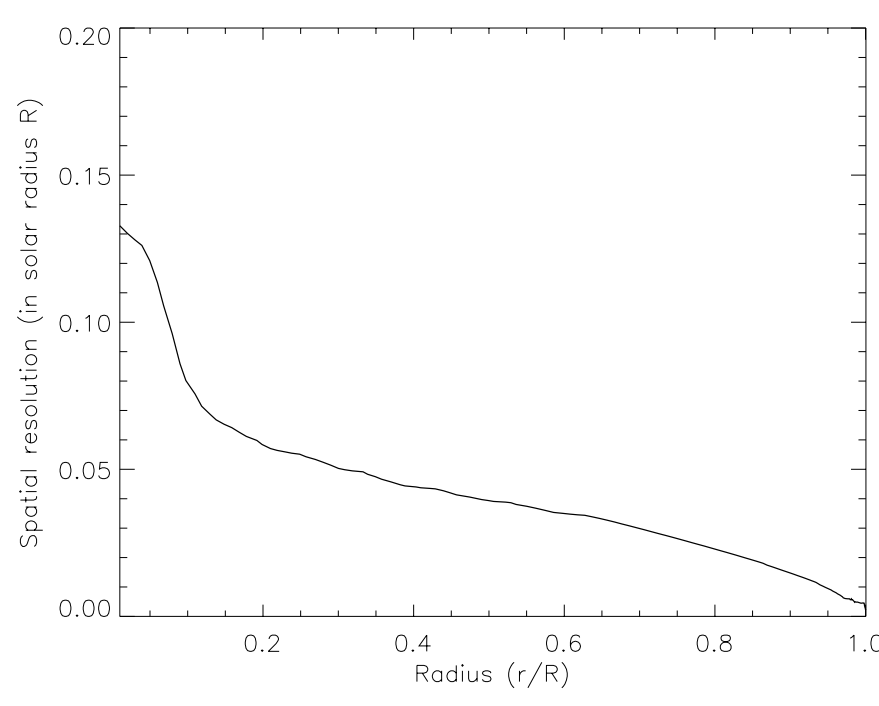

Fig. 3. Spatial resolution of the solution for test 1

Concerning the penalty function, we know that the mesh has four times the optimal number of points, so we will apply a fourth difference smoothing function $H$. To do this, we have to minimize the quadratic difference between the value of the solution at one point $f_{i}$ and the average value of the four surrounding points

$$
\left|f_{i}-\frac{f_{i-2}+f_{i-1}+f_{i}+f_{i+1}+f_{i+2}}{5}\right|^{2} \quad i=1, \ldots, N
$$

Let we now consider the weighting of the penalty function. When the RLS technique was explained, a constant weighting parameter $\gamma$ was defined, so the smoothing constraint is applied in the same way to all the solution. But then the weighting parameter is mainly determined by the result at the points that have a higher sensitivity to the kernels and, hence, the smoothing at other points can be dragged. To avoid this problem, we have applied a different weighting parameter $\gamma_{i}$ at each point in the solution. We give now the general expression for the penalty function and then describe how our method compute $\gamma_{i}$.

In general, the minimization of relation (16) can be expressed by a matrix $H=Q^{\mathrm{T}} Q$, where $Q$ is defined by

$$
Q=\left(\begin{array}{c}
\gamma_{1} \\
\gamma_{2} \\
\gamma_{3} \\
\cdot \\
\cdot \\
\gamma_{N}
\end{array}\right)^{\mathrm{T}}\left(\begin{array}{cccccccc}
\frac{4}{5} & -\frac{1}{5} & -\frac{1}{5} & 0 & . & . & . & . \\
-\frac{1}{5} & \frac{4}{5} & -\frac{1}{5} & -\frac{1}{5} & 0 & . & . & . \\
-\frac{1}{5} & -\frac{1}{5} & \frac{4}{5} & -\frac{1}{5} & -\frac{1}{5} & 0 & . & . \\
0 & -\frac{1}{5} & -\frac{1}{5} & \frac{4}{5} & -\frac{1}{5} & -\frac{1}{5} & 0 & . \\
\cdot & \cdot & . & \cdot & \cdot & \cdot & \cdot & . \\
\cdot & . & . & . & . & -\frac{1}{5} & -\frac{1}{5} & \frac{4}{5}
\end{array}\right)
$$

in which case

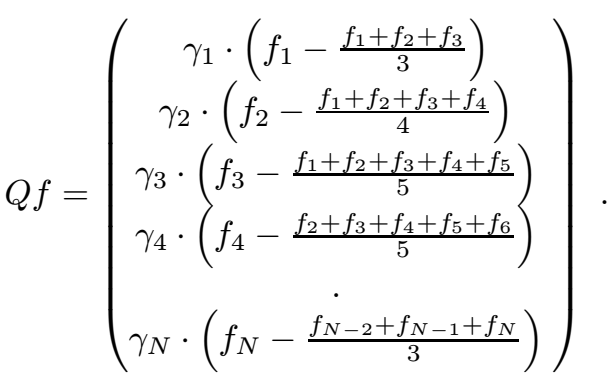

Notice that since the $\gamma_{i}$ are included in $Q$, and hence in $H$, Eqs. (4) and (5) are replaced by

$$
\begin{aligned}
& f^{\text {est }}=\left(A^{\mathrm{T}} A+H\right)^{-1} A^{\mathrm{T}} D \\
& \varepsilon^{\text {est }}=\left(A^{\mathrm{T}} A+H\right)^{-1} A^{\mathrm{T}} \Sigma .
\end{aligned}
$$

The parameters $\gamma_{i}$ have been calculated following a method developed by Ruiz Cobo \& del Toro-Iniesta (1994) of inverting the Stokes polarization line profiles. The matrix $A$, defined in Eq. (2), can be written by using Spectral Value Decomposition (SVD) as

$A=U \cdot \Lambda \cdot V^{\mathrm{T}}$

where $\Lambda$ is a diagonal matrix that contains the eigenvalues of $A$. $U$ and $V$ are orthonormal matrixes, with $V$ containing the eigenvectors of $A$.

Each point $r_{i}$ of the mesh has its own contribution to the eigenvalue matrix $\Lambda$. Let us introduce the matrixes $\Lambda_{i}$ defined by

$\Lambda_{i}=\Lambda \cdot W_{i}^{\mathrm{T}} \cdot V$

where the elements $w_{i}^{j k}$ of $W_{i}$ are related to the elements $v^{j k}$ of $V$ by the relation

$w_{i}^{j k}=v^{j k} \delta_{j i} \quad i=1, N$.

It is straightforward to demonstrate that

$\Lambda=\sum_{i=1}^{N} \Lambda_{i}$

The contribution of each point $r_{i}$ is taken as the maximum value of the corresponding diagonal of the matrix $\Lambda_{i}$, and the weighting factors $\gamma_{i}$ are given by

$\gamma_{i}=c \cdot \max \left(\Lambda_{i}\right) \quad i=1, \ldots, N$,

where $c$ is just a proportionality factor. In Fig. 4, the contributions of points for test 1 are presented. As expected, the contributions for outer points are higher than for inner points.

In summary, we have obtained a method, similar to RLS, that smoothes each point in the solution independently. The form of the smoothing function is given by the spatial resolution, which also gives the distribution of points in the inversion mesh. The only free variable of the method is the proportionality factor $c$, introduced in Eq. (23), which will play the role of the trade-off parameter between error magnification and accuracy of the solution. 


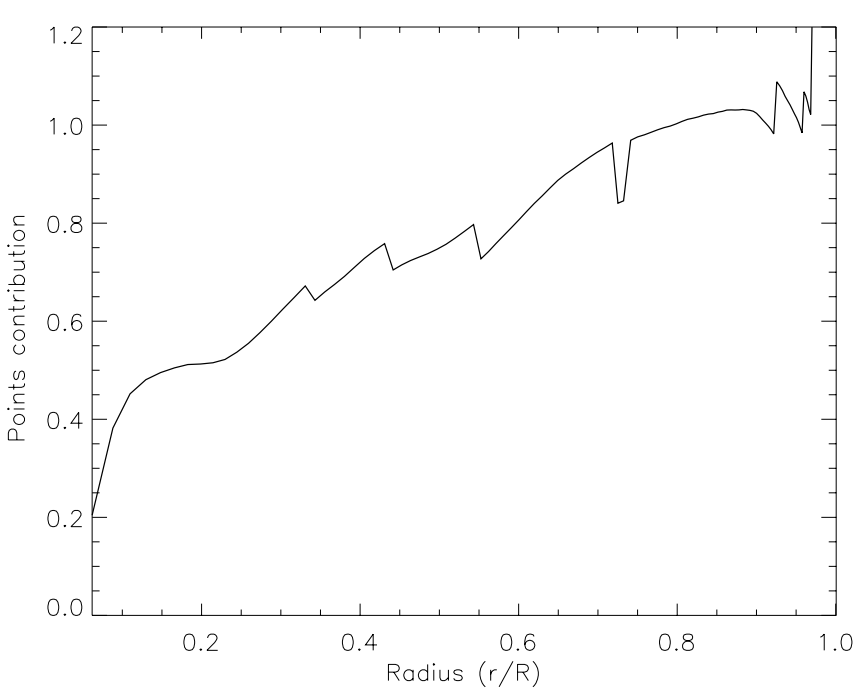

Fig. 4. Point contributions for test 1

\subsection{Solution for test 1}

Here, we are going to see the performance of the method by inverting artificial data corresponding to test 1 . To obtain the solution, we give different values to $c$, the only free variable of the method, and calculates $\chi^{2}$ and the error propagation for each solution. The error propagation of the solution is defined as a quadratic average over all the points of the mesh. Figure 5 shows $\chi$ versus the error propagation for different values of $c$. The curve has two trends: for small values of $c, \chi^{2}$ is almost invariant, while the error decreases very fast. For high $c$ values the invariant behaviour is obtained for the error propagation (oversmooth solution). We have chosen as the optimal $c$ the one where the two different trends concur (the "elbow" of the curve).

Figure 6 shows the solution obtained by our code for test 1 . The result is quite good and fits the actual solution below the error level.

OMD is a mixture of standard methods and is neither the fastest nor the slowest one. Over $75 \%$ of computing time is dedicated to obtain the spatial resolution, while $20 \%$ is used by the inversion analysis. The rest of the time is used to read files. The time ratio between RLS (the fastest), OMD and OLA is, approximately $1: 12: 40$ for test 1.

\section{Application to structural inversions}

We have seen how OMD works when only one function, $f(r)$, is inverted. In this section, we extent the method to the case where two functions $f_{1}(r)$ and $f_{2}(r)$ are to be determined. An important example of this kind of problem is represented by the inversion for solar structure, in our case sound speed $c$ and density $\rho$. These are commonly based on the linearization of the equations of stellar oscillations around a reference model (e.g. Gough \& Kosovichev 1988;

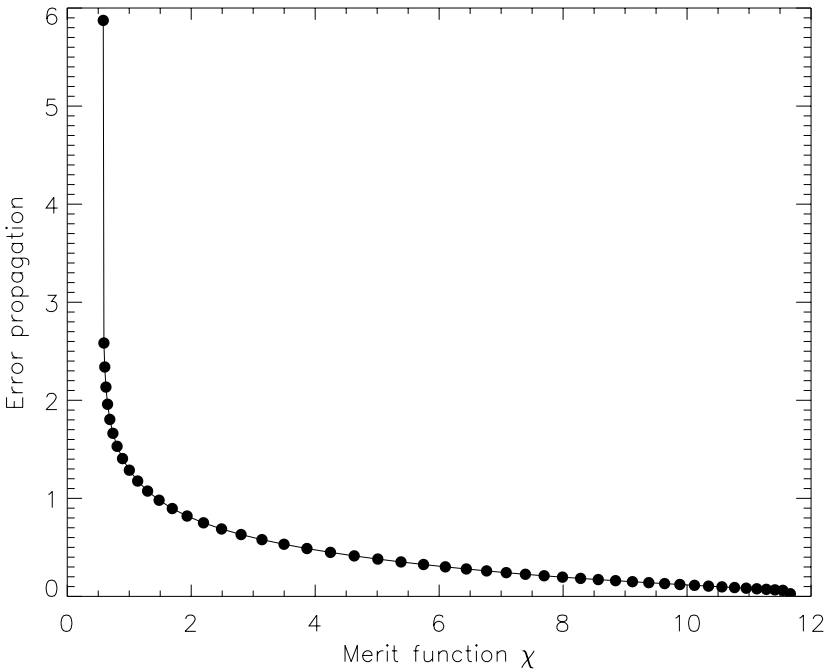

Fig. 5. $\chi$ versus error propagation for different values of the trade-off parameter $c$

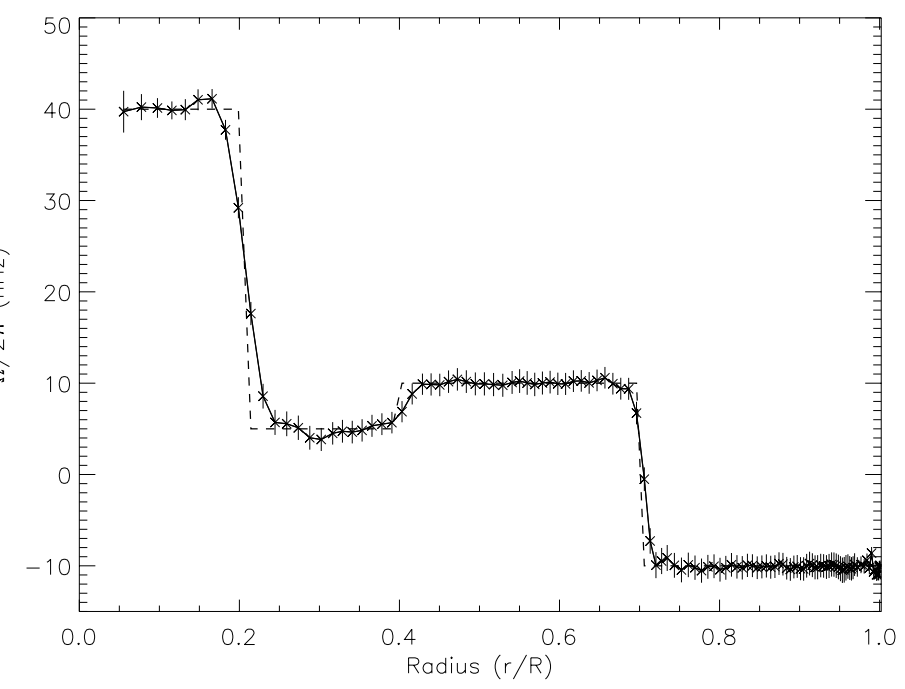

Fig. 6. Result for test 1. The dashed line represent the actual solution and solid line shows the inverted solution with its error bars

Dziembowski et al. 1990; Gough \& Kosovichev 1990). Here, structural differences between the actual Sun and the model are linearly related to differences between the observed frequencies and those calculated using the model. This relation is obtained by using a variational formulation for the frequencies of adiabatic oscillations. A general relation for frequency differences is given by

$$
\begin{aligned}
\frac{\delta \omega_{i}}{\omega_{i}} & =\int_{0}^{R}\left[K_{c, \rho}^{i}(r) \frac{\delta c}{c}(r)+K_{\rho, c}^{i}(r) \frac{\delta \rho}{\rho}(r)\right] \mathrm{d} r \\
& +\mathcal{E}_{i}^{-1} F(\omega)+\epsilon_{i},
\end{aligned}
$$

where $\delta \omega_{i}$ (with relative error $\epsilon_{i}$ ) is the difference in frequency of the $i^{\text {th }}$ mode between the actual Sun and the model. The functions $\delta c / c$ and $\delta \rho / \rho$ are the parameters 
to be inverted. $K_{c, \rho}^{i}(r)$ and $K_{\rho, c}^{i}(r)$ are known functions, called kernels, that relate the changes in frequency to the changes in the model. The term $\mathcal{E}_{i}^{-1} F\left(\omega_{i}\right)$ in (24) takes into account the uncertainties at the solar surface, mainly due to the incorrect modelling of the outer part of the convection zone, non-adiabatic effects and the omission of some surface terms. Following standard procedures (Dziembowski et al. 1990), we represent $F\left(\omega_{i}\right)$ as a Legendre polynomial function. $\mathcal{E}_{i}$ is the inertia of the mode, normalized by the photospheric amplitude of the displacement and divided by the inertia that a radial mode of the same frequency would have (see Gough \& Thompson 1991 for further details).

With small changes in the formulation developed in Sect. 2, we can solve Eq. (24) by using OMD. The main problem in this kind of inversion is that one of the functions could be more sensitive to the data set than the other one. That is our case: p-mode data (the ones we have at present) are very sensitive to sound speed variations, so the contribution of this function (and its kernels) to the inversion will be higher than the one for density. It means that the weight given to the penalty function and the distribution of points in the inversion mesh will be different for the two functions.

To test the inversion method, instead of the difference between the actual Sun and a model, we have considered two models. In particular, test 2 uses two models from Christensen-Dalsgaard \& Berthomieu (1991) that differ in the opacities. One of these, the reference model, uses the tables of Cox \& Tabor (1976), while the other one uses the opacities from Lebbreton \& Maeder (1986). Both models have the equation of state of Eggleton et al. (1973) and the parameters of nuclear energy generation from Parker (1986). The abundance of heavy elements is, in both cases, $Z=0.02$. From these models, we obtain the oscillation frequencies for degrees in the range $0 \leq l \leq 140$ (ChristensenDalsgaard \& Berthomieu 1991). The mode set and errors are taken from actual observations.

The only significant difference in the inversion procedure is in the spatial resolution analysis. Because there are two different kernels sets, Eq. (14) is replaced by

$b=\left(C^{\mathrm{T}} C+C^{\prime \mathrm{T}} C+\lambda I\right)^{-1} C^{\mathrm{T}} y$

such that when the linear combination of one kernel set, with covariance matrix $C$, fits a target sine of a given spatial frequency, the combination of the kernels associated with the other parameter, with covariance matrix $C^{\prime}$, are zero, that is $\left|C^{\prime} b\right|^{2} \leq \mathrm{e}^{\prime 2}$.

Then the spatial resolution is obtained in the same way as explained for one function. In Fig. 7, the spatial resolution for density and sound speed, using the test 2 data set, are presented. In this case, where only p-modes are used, there are no significant differences between the two distributions.

However, since p-mode data are more sensitive to sound speed variations than to density variations, the

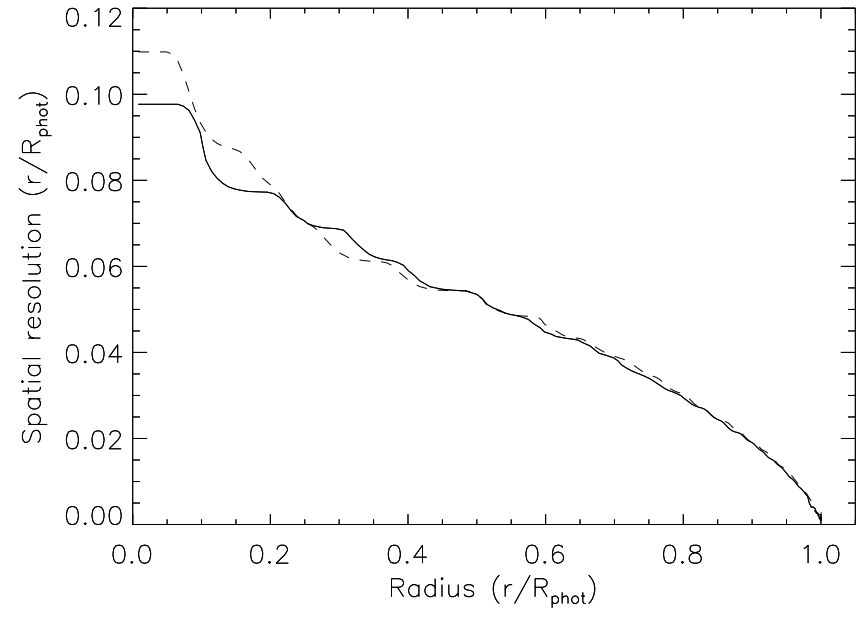

Fig. 7. Spatial resolution in sound speed (solid line) and density (dashed lines) for test 2

point contributions to the solution and hence the weighting parameters $\gamma_{i}$ are different. This can be seen in Fig. 8. For sound speed the contribution of the external points is larger but is not so pronounced for the density.

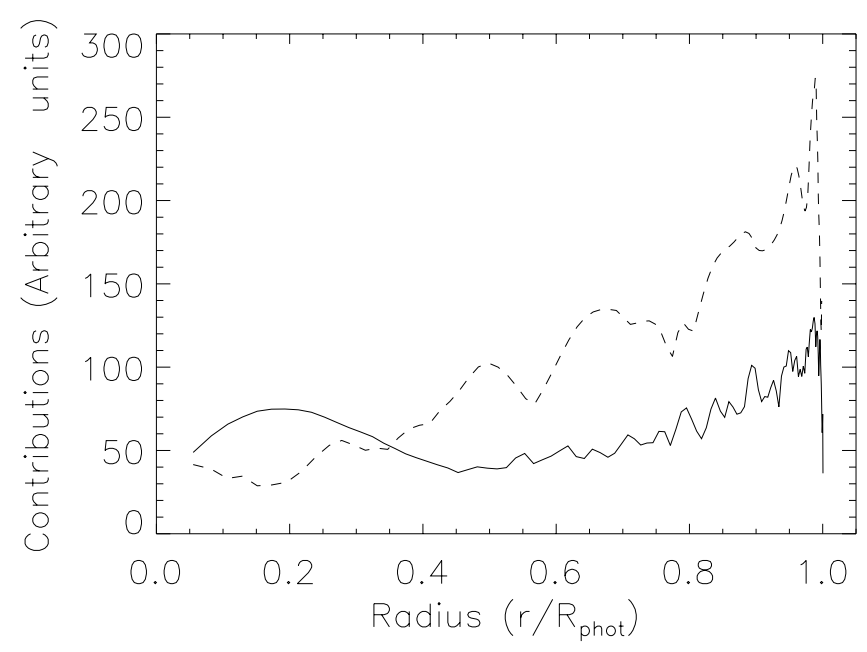

Fig. 8. Point contributions in the case of test 2 for sound speed (dashed lines) and density (solid line)

Figure 9 shows the solution obtained for test 2. The result is quite good, although the discrepancy between the actual solution and the inverted one, as well as the error level, is higher in density because $p$ modes are more sensitive to sound speed variations.

The precision of the result can also be tested in a different way, by means of evaluating the averaging kernels obtained in the inversion. We know that the solution vector $f^{\text {est }}$ is given by Eq. (17). Data vector $D$ is defined by $D=A f$, where $f$ is the actual solution, so that

$f^{\text {est }}=\left(A^{\mathrm{T}} A+H\right)^{-1} A^{\mathrm{T}} A f$. 


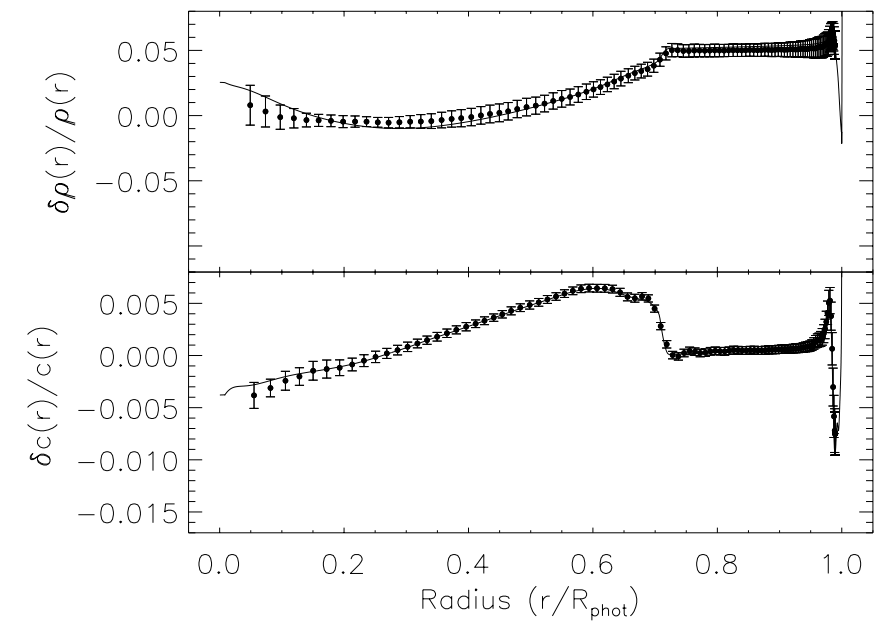

Fig. 9. Density and sound speed results for test 2. The solid line represent the actual solution and filled circles with their error bars, the inverted one

Averaging kernels for each point corresponds to the rows of matrix $\left(A^{\mathrm{T}} A+H\right)^{-1} A^{\mathrm{T}} A$, and give an idea of how the inversion method filters the actual solution $f$. Our solution $f^{\text {est }}$ would be exact if the average kernels were Dirac delta functions of unit area for the parameter we are inverting (i.e. sound speed) and zero for the other parameter (i.e. density). Figures 10a and 10b show averaging kernels when sound speed and density are inverted. It can be seen that the averaging kernels obtained by our method are good, especially for sound speed. Results for density are fairly good, and hence it can be expected that the solutions for this parameter will be reasonably accurate.

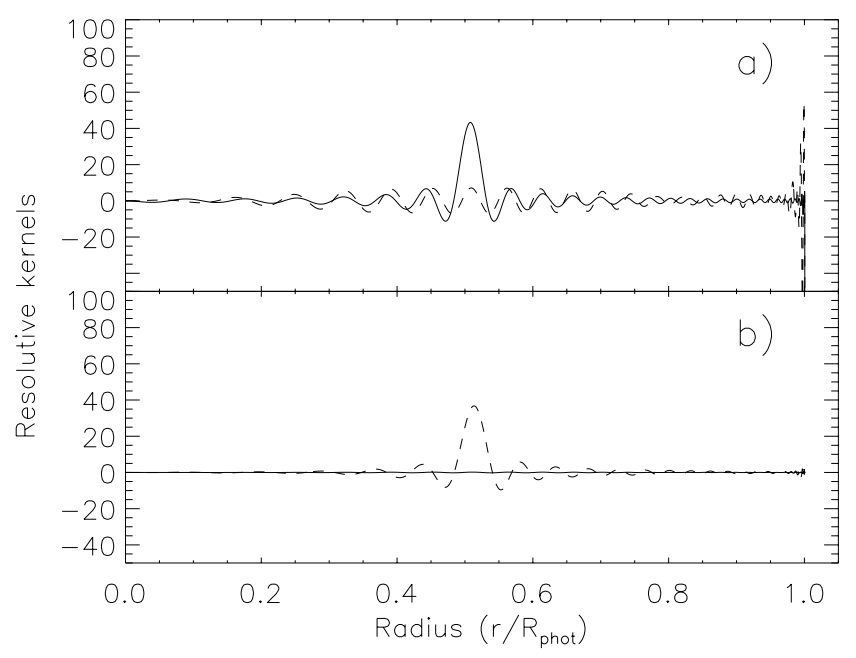

Fig. 10. a) Averaging kernel at $r / R=0.5$ when density is inverted. In dashed and solid lines the components associated to sound speed and density, respectively, are presented. b) The same as a) but when sound speed is inverted
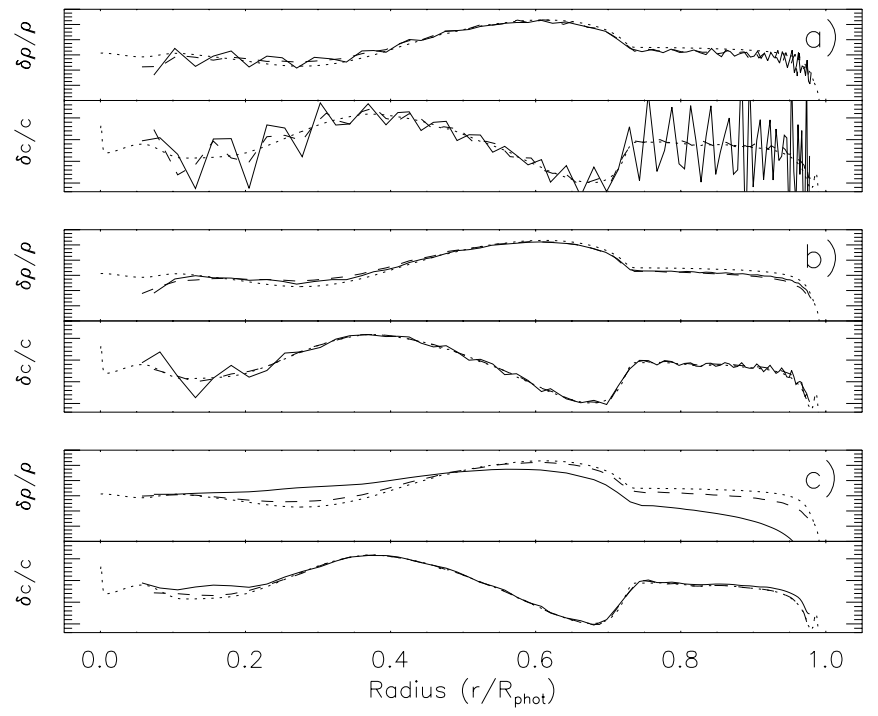

Fig. 11. Behaviour of the solution for three different values of the proportionality factor, $c$. The actual solution is presented in dotted lines. The result with OMD is shown in dashed lines and the one with the regularization technique in solid line

A comparison between OMD and a regularization technique with only one smoothing parameter $\gamma$ and a mesh with equally-spaced points has been done. Results are presented in Fig. 11. Each panel is associated with a different value of $c$ (for OMD) and $\gamma$ (for RLS) in the inversion. Figure 11a shows that with OMD (dotted line), the response of density and sound speed results to the smoothness is equivalent, but with the other technique (solid line), density is smoothed before sound speed. In Fig. 11b, the solution for density is very good using the two methods, but the one for sound speed is still undersmooth with the standard technique. To obtain a non-oscillatory result in sound speed with the standard method, it is necessary to increase the weight of the smoothness, but this means that the density solution will be oversmooth (see Fig. 11c). It can be seen that these problems are solved with OMD. The possibility of applying different weights to the penalty function, not only in radius but also in each function to be inverted, is a great advantage of OMD.

It is important to note that when sound speed and density are inverted, it is unnecessary to assume that the equation of state is known. This is not the general case, for instance, if the density is estimated by inverting $\rho$ and the helium abundance $Y$. In this case, an equation of state must be assumed in order to obtain the kernels (e.g. Basu et al. 1996) and hence the result can be affected by this.

\section{Conclusions}

We have developed a new inversion method (OMD) suitable for helioseismology. OMD calculates the spatial resolution of the solution in order to build an optimal inversion 
mesh and select the smoothing criterion. The weight of such a smoothing function, at each tabular point, is given by the SVD analysis of the contribution of each point in the mesh to the solution. The method have been tested by applying it to artificial data. We have found that the solution fits the actual one within errors for all the cases considered. Also the behaviour of the averaging kernels illustrates the accuracy of the results.

Although the method is suitable for inversions of only one function, such as the rotational one used for test 1 , it shows its advantage when two functions are inverted, especially because the weight of the smoothing function is applied in a different way for each radial point and each function to be inverted.

The fact that we are able to invert sound speed and density at the same time, is very important because the inversion is independent of the equation of state. Consequently, the solution obtained with OMD is more robust in this sense. Accuracy and precision are not the only advantages of OMD. Our method is also computationally faster than OLA or similar techniques.

Acknowledgements. We thank D.O. Gough for his help during the development of OMD technique. This work has been made possible thanks to the financial support of the Spanish DGICYT under grants ESP90-0969 and PB91-0530.

\section{References}

Backus G., Gilbert F., 1968, Geophys. J. R. Astron. Soc. 16, 169

Backus G., Gilbert F., 1970, Phil. Trans. Ser. A 266, 123
Basu S., Christensen-Dalsgaard J., Schou J., Thompson M.J., Tomczyk S., 1996 (submitted to ApJ Letters)

Christensen-Dalsgaard J., Berthomieu G., 1991, in Solar Interior and Atmosphere, Cox A.N., Livingston W.C., Matthews M.S. (eds.), p. 401

Cox A.N., Tabor J.E., 1976, ApJS 31, 271

Craig I.J.D., Brown J.C., 1986, Inverse Problems in Astronomy: A Guide to Inversion Strategies for Remotely Sensed Data. Bristol: A. Hilger

Dziembowski W., Pamyatnykh A.A., Sienkiewicz R., 1990, MNRAS 244, 542

Eggleton P.P., Faulkner J., Flannery B.P., 1973, A\&A 23, 325

Gough D., Kosovichev A.G., 1988, in Seismology of the Sun and Sun-like stars, ESA SP-286, p. 195

Gough D.O., Kosovichev A.G., 1990, in Inside the Sun, Berthomieu G. \& Cribier M.(eds.). Dordrecht, Kluwer, p. 327

Gough D.O., Thompson M.J., 1991, in Solar Interior and Atmosphere, Cox A.N., Livingston W.C., Matthews M.S. (eds.), p. 519

Lebbreton Y., Maeder A., 1986, A\&A 161, 119

Parker P.D., 1986, in Physics of the Sun, Vol. 1, Sturrock P.A., Holzer T.E., Mihalas D., Ulrich R.K. (eds.). Reidel, Dordrecht, Holland, p. 15

Pijpers F.P., Thompson M.J., 1994, A\&A 281, 231

Ritzwoller M.H., Lavely E.M., 1991, ApJ 369, 557

Ruiz Cobo B., del Toro Iniesta J.C., 1994, A\&A 283, 129

Thompson M.J., 1995, Inv. Prob. 11, 709 